\title{
Inherent Promotion of Ionic Conductivity via Coherent Vibrational Strong Coupling of Water
}

\author{
Tomohiro Fukushima, Soushi Yoshimitsu, Kei Murakoshi \\ Department of Chemistry, Faculty of Science, Hokkaido University, \\ Sapporo, Hokkaido, 060-0810, Japan.
}

\begin{abstract}
The vibrational strong coupling, where vibrational energy states of molecules and an optical cavity mode are hybridized, is an emerging method for the modification of ground-state thermochemical reactivity of molecules. Here, we report that vibrational strong coupling promotes ionic conductivity of aqueous electrolyte. A Fabry-Pérot cavity were fabricated for the spectroscopic analysis and electrochemical impedance measurements of electrolyte solution. We observed the splitting of vibrational state of water in optical cavity with the Rabi splitting energy of $760 \mathrm{~cm}^{-1}$ by matching cavity mode frequency to $\mathrm{OH}$ stretching mode frequency of water molecules. From the electrochemical impedance measurements, we discovered the modulation of relative dielectric constants and one-fold promotion of proton conductivity of the aqueous electrolyte. This study demonstrates that the use of vibrational strong coupling system for the promotion of ionic conductivity utilized for the electrochemical energy conversion system without light pumping.
\end{abstract}


The strong coupling of energy state of molecules to the optical cavity vacuum fields is an emerging method for modulation of molecular or material properties, such as modification of energy level via coherent interaction between single molecules. ${ }^{1-3}$ On one hand, electronic excitation of energy states of molecules are coupled to the electromagnetic fields and giving the unusual photochemical dynamics $^{4,5}$ or coherent electronic transport. ${ }^{6-8}$ On the other hand, vibrational level of molecules can be coupled to the vacuum fields of optical cavity for the formation of vibrational strong coupling state, ${ }^{9-12}$ leading to the modification of chemical landscapes for the reactivity modulation. ${ }^{13-16}$ That is related to the classical concept of "vibrational excitation for bond-selective chemical reactions"17 even without light pumping. The utilization of vibrational strong coupling can open up the opportunity to modulate the chemical dynamics, which plays an important role in chemistry. In particular, hydrogen bonding dynamics of water plays a vital role for the physicochemical properties of water. ${ }^{18-22}$ Even in the static condition, the proton is transported by sequential orientations of water molecules occurring along the hydrogen bond network as known as Grotthuss mechanism. ${ }^{23,24}$ The promotion of the proton conductivity is important topics in electrochemistry. ${ }^{25-29}$ However, there are no demonstration on the utilization of vibrational strong coupling on the ionic transport or electrochemistry.

Herein, we demonstrate the vibrational strong coupling of water to promote the ionic conductivity. We observed Rabi splitting of vibrational energy state in water and acidic solution. Interestingly, vibrational strong coupling modulates the dielectric constant and even one-fold increase of the proton conductivity. The origin of the promotion of the proton conductivity by vibrational strong coupling is 
discussed.

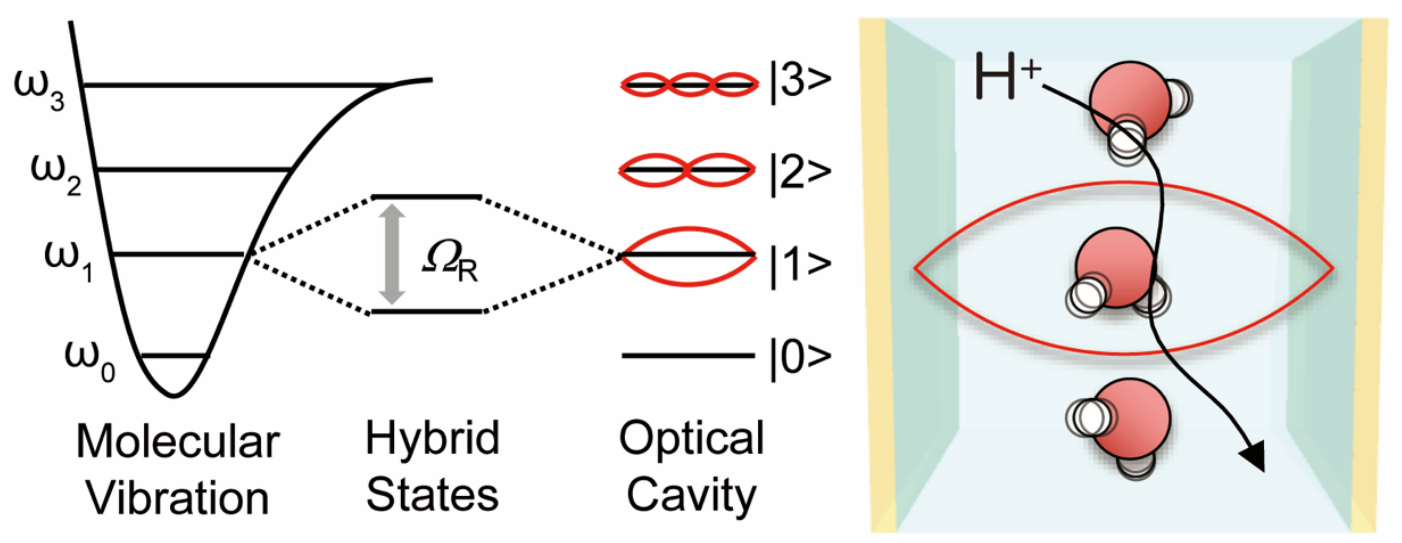

Figure 1. Schematic illustration of vibrational strong coupling by hybridization of molecular vibration and optical cavity mode. The demonstration of proton conductivity within the cavity.

A Fabry-Pérot cavity consisting of two parallel mirrors separated by Mylar film was prepared as shown in Figure S1. Mirrors were fabricated by deposition of $20 \mathrm{~nm} \mathrm{SiO}_{2}$ and $8 \mathrm{~nm} \mathrm{Au}$ on $2 \mathrm{~mm} \mathrm{CaF}_{2}$ window. Mirrors were sandwiched with home-made cell, and cavity thickness was tuned by screw. The holes for the liquid insertion were used for the positioning of two-electrode setup of Pt wires for electrochemical measurements. The homogeneity of the cavity thickness was checked by interference structural color of the optical cavity. It is essential to position two mirrors parallelly of a Fabry-Pérot cavity in order to observe the modulation of electrochemical properties of electrolyte solution as described hereafter.

A IR transmission spectrum of the air-filled Fabry-Pérot cavity is shown in Figure S2. The 
spectroscopy showed progression of optical cavity modes as a result of interference characteristic of optical cavity. Optical cavity modes can be determined by the fringe in peak-to-peak separation of cavity modes. Cavity thickness can be controlled between the range of $5 \mu \mathrm{m}$ to $100 \mu \mathrm{m}$ by using Mylar films: longer cavity thickness leads to the narrower free spectral ranges and shorter cavity thickness leads to the wider free-spectral-ranges. The detuning of the screw can optimize the optical mode of the cavity. In our case, spacer was eliminated for the achievement of thinner condition of optical cavity cell. The electrolyte solution was placed between mirror without spacer, and cavity thickness was tuned to the lower order mode with the cavity length less than $5 \mu \mathrm{m}$. Importantly, quality factor of cavity mode is similar each other independent to the mode number of cavity mode, suggesting that the quality of the cavity is maintained throughout the experiments.

Vibrational state of water in optical cavity was evaluated by detuning of cavity thickness. Water was sandwiched with the two parallel mirrors and IR spectra was evaluated as shown in Figure 2a. The optical cavity mode frequency was detuned by controlling cavity thickness. To characterize the cavities, we extrapolate the coupled cavity mode position from one of the uncoupled cavity modes where water shows no absorption bands. When the cavity mode was matched to the $\mathrm{OH}$ stretching mode frequency, original absorption peak was absent and the two unique peaks, which can be assigned to the vibropolaritonic states, were observed. This tendency is consistent with the previous reports for the vibrational strong coupling of water. ${ }^{30-34}$ Cavity mode was detuned from $\mathrm{OH}$ stretching mode frequency to evaluate the anti-crossing behavior of upper polariton branch and lower polariton branch 
as shown in Figure 2b. We observed Rabi splitting behavior with the first order modes of optical cavity without the saturation by intense $\mathrm{OH}$ stretching absorption band. Rabi splitting energy was estimated when the cavity mode is tuned to the $\mathrm{OH}$ stretching mode, and the observed Rabi splitting energy was $760 \mathrm{~cm}^{-1}$. In case with the strong coupling condition, light is confined in the cavity and coherent interaction was observed, which means that Rabi splitting energy, $\hbar \omega_{\mathrm{R}}$, is larger than sum of photon loss rate of a cavity, $\kappa$, and dephasing rate of $\mathrm{OH}$ stretching of water molecules, $\gamma \cdot{ }^{35-37}$ Fullwidth at half maximum (FWHM) of $\mathrm{OH}$ stretching mode, which corresponds to the dephasing rate, was $510 \mathrm{~cm}^{-1}$. FWHM of cavity modes, which corresponds to the photon loss rate of a cavity, were $200 \mathrm{~cm}^{-1}$. The comparison between Rabi splitting energy and photon loss rate and dephasing rate can guarantee the vibrational strong coupling state of water molecules in cavity even in the presence of electrolyte. ${ }^{30-34}$
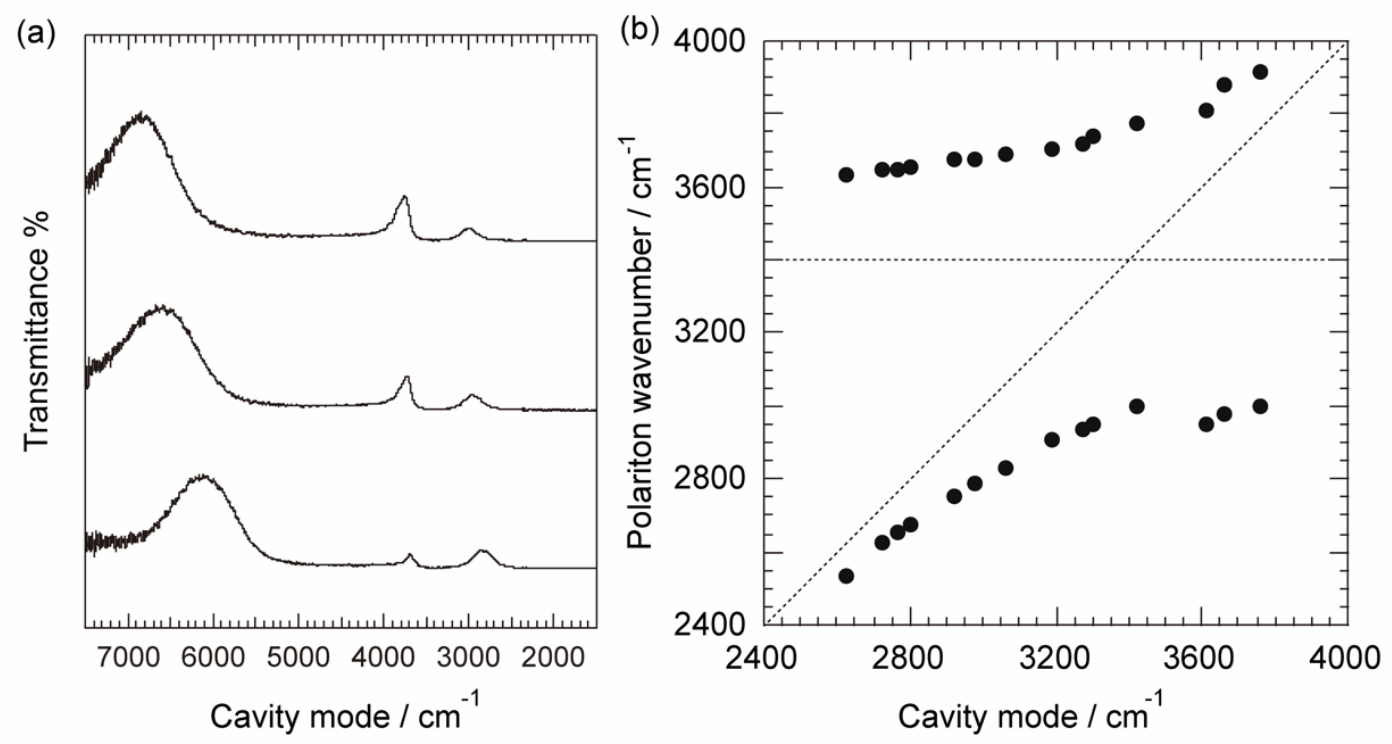

Figure 2. (a) IR spectra of water in cavity. (b) The anti-crossing behavior of water in optical cavity. 
We evaluated the electrochemical properties of aqueous electrolyte $0.01 \mathrm{M} \mathrm{HClO}_{4}$ aqueous solution under vibrational strong coupling. Electrochemical impedance measurements were conducted by using two platinum electrodes. We first evaluated the effect of cavity formation by using non-sputtered $\mathrm{CaF}_{2}$ cell systems. Figure 3a shows the typical Cole-Cole plot for in the presence and absence of cavity effect. In both case, equivalent circuit composed of a series of resistance and two sequential Randles circuit, which are parallel circuit of resistance and capacitance. A resistance can be assigned to the electrical resistance of the circuit. One Randles circuit can be assigned to the electrolyte and the other Randles circuit can be assigned to the electrode-electrolyte interface. Importantly, we observed the decrease in resistance and similar value for capacitance in case with the same electrochemical cell setup by using cavity type cell. In this case, cell constant of the optical cavity cell is similar each other, therefore, the difference in the resistance and capacitance are derived from the ionic conductivity and relative dielectric constant. The resistance and capacitance of $0.01 \mathrm{M} \mathrm{HClO}_{4}$ electrolyte for the cavity cell solution were $0.720 \mathrm{M} \Omega$ and $0.198 \mathrm{pF}$, respectively. Whereas the resistance and capacitance of $0.01 \mathrm{M} \mathrm{HClO}_{4}$ electrolyte for the non-cavity cell were $3.59 \mathrm{M} \Omega$ and $0.182 \mathrm{pF}$, respectively. These observations suggest that vibrational strong coupling of $\mathrm{OH}$ stretching mode in water molecules can attributes to the decrease in the resistance and promotion of ionic conductivity.

We observed that ionic conductivity of water can be controlled by the cavity thickness by tuning the cavity mode. Ionic conductivity of $0.01 \mathrm{M} \mathrm{HClO}_{4}$ electrolyte showed $0.013 \mathrm{~S} \mathrm{~cm}^{-1}$ in the presence of cavity effect with off-resonance geometry condition. Surprisingly, tuning of cavity mode to the $\mathrm{OH}$ 
stretching frequency promoted ionic conductivity. Ionic conductivity of $0.01 \mathrm{M} \mathrm{HClO}_{4}$ electrolyte showed $0.19 \mathrm{~S} \mathrm{~cm}^{-1}$ in the presence of cavity effect with on-resonance geometry condition, which is one-fold higher value than the same geometry in off-resonance condition. On the other hand, the relative dielectric constants showed similar values of 82 weakly reached to the 120 for on-resonance condition. In addition, we observed isotope effect on the ionic conductivity with cavity effect. The resonant cavity modes of the vibrational strong coupling of water isotopes are different because the resonant wavelength between the $\mathrm{OH}$ stretching and the $\mathrm{OD}$ stretching modes are different due to the mass of the hydrogen isotopes. Figure $\mathrm{S} 3$ shows the ionic conductivity of $\mathrm{HClO}_{4}$ and $\mathrm{DClO}_{4}$ with respect to the cavity length. In case with the proton conductivity, the maximum enhancement on the ionic conductivity showed $0.19 \mathrm{~S} \mathrm{~cm}^{-1}$ with the cavity length of the $1.2 \mu \mathrm{m}$. In case with the deuteron conductivity, the maximum enhancement on the ionic conductivity showed $0.14 \mathrm{~S} \mathrm{~cm}^{-1}$ with the cavity length of the $1.5 \mu \mathrm{m}$. The feasible point of the cavity-enhanced ionic conductivity is that the relative ionic conductivity can be controllable by selective coupling of molecular vibration to the cavity mode. The observed cavity-dependent behavior on ionic conductivity depending on the behavior excludes the possibility of the surface-enhanced ionic transport by the formation of diffusion layer in micro cavity, ${ }^{38}$ or structured water formation from the wall of microchannels. ${ }^{39,40}$ The observed electrochemical behavior is inherently different from the previously reported electrochemical phenomena in microchannels. 

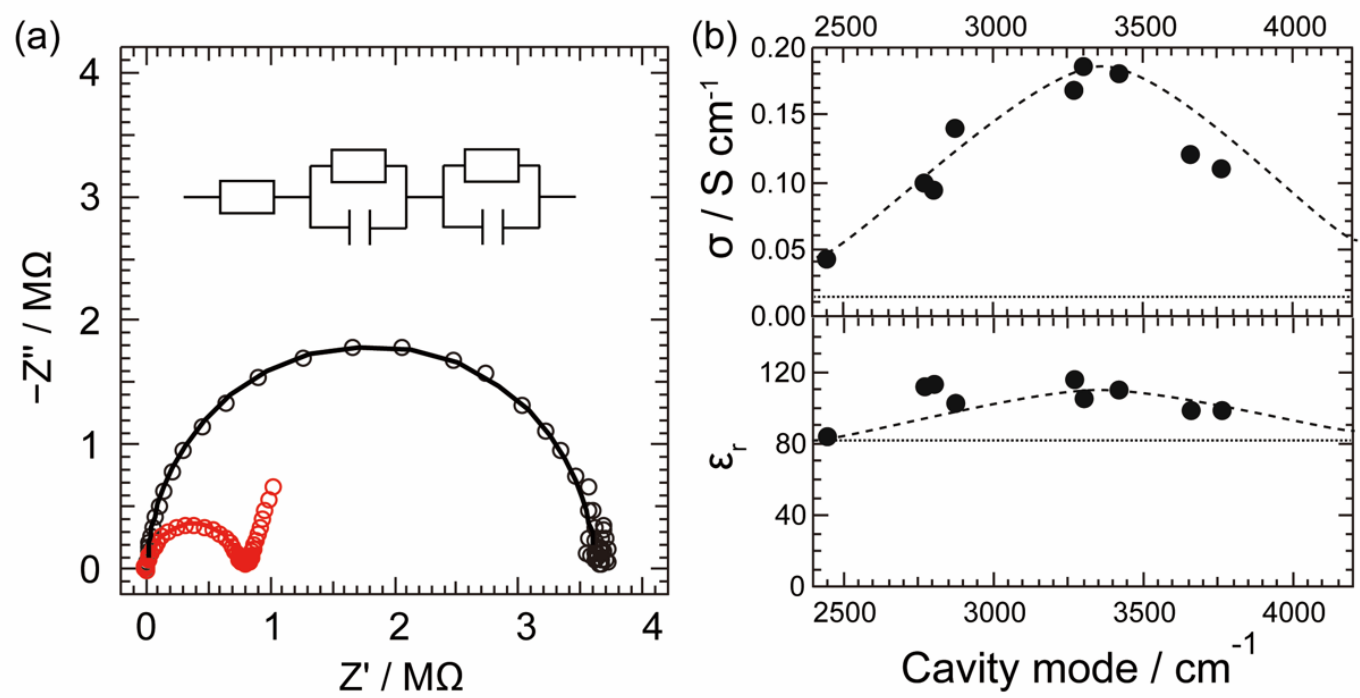

Figure 3. (a) Cole-Cole plot of $0.01 \mathrm{M} \mathrm{HClO}_{4}$ with cavity effect (red) and without cavity effect (black) and equivalent circuit (inset). (b) The dependence of conductivity and dielectric constant to the optical cavity mode frequency. Dotted straight lines are ionic conductivity and dielectric constant of bulk electrolyte.

In addition, we successfully observed inherent cavity effect on the ionic conductivity of electrolyte solution. In case with the tetrabutylammonium hexafluorophosphate $\left(\mathrm{TBAPF}_{6}\right)$ acetonitrile solution, vibrational strong coupling of acetonitrile was not observed from the IR spectra as shown in Figure S4. This is due to the weak oscillator strength of single acetonitrile molecule compared to the one of single water molecule. We cannot observe any perturbation of electrochemical properties of TBAPF 6 solution even in the presence of cavity effects as shown in Figure S5. Whereas inherent promotion of the ionic conductivity was observed in case with the aqueous solution. We observed small increase in the ionic conductivity in $\mathrm{KCl}$ aqueous electrolyte over 5 times, which is smaller value compared to the 
cavity effect in $0.01 \mathrm{M} \mathrm{HClO}_{4}$. The observation can be due to the vehicle mechanism, where hydrated $\mathrm{K}^{+}$and $\mathrm{Cl}^{-}$diffuse in the solution as an ionic carrier. Therefore, the major effect of vibrational strong coupling was not observed compared to the proton carrier with the Grotthuss mechanism. For example, the self-ionization of water to produce the $\mathrm{H}^{+}$as the ionic carrier and promotion. As a proof of concept, we observed the promotion of the ionic conductivity of milli-Q water over one-fold as shown in Figure S6. From these results, we can expect that vibrational strong coupling can modify the ground state proton transfer step in the proton conductivity.

We would like to discuss the origin of the promotion of the ionic conductivity. In general, proton conductivity are related to the dynamic transfer of hydrogen bonding, ${ }^{25-29}$ and dielectric constantans are related to the density of molecules and molar polarization. ${ }^{41-43}$ Our finding reported here are consistent with the fact that coupling a vibrational mode to a cavity mode alters the potential energy landscape causing modification of ground-state reactivity, which was also related to the proton transfer reaction. Generally ionic conductivity, $\sigma$, can be modelled via Nernst-Einstein equation and relative dielectric constant, $\varepsilon_{\mathrm{r}}$, can be represented by Debye's equation. We presumed that the strong coupling facilitates on the (1) vibrational structural change (mode softening/hardening) of water molecules and (2) coherence between individual vibrational mode in water molecules. In addition, the energy of the ground-state did not change energy state does not change, which is often observed for the deep strong coupling.

We would like to first discuss the effect of vibrational strong coupling on the aspect of mode 
softening and hardening. We assumed that the vibrational strong coupling can modulate the enthalpy terms resulted from force constants without changing the dissociation energy, and negligible difference in the entropy term for liberation, translation, vibration, solvation. We note that the energy level of the $\mathrm{OH}$ stretching mode is same because ground-sate modulation cannot be observed for even strong coupling region (detailed discussion are described in Supporting Information). Water dimer structure is modeled for the proton transfer as shown in Figure S7a, and potential energy curves were calculated as shown in Figure S7b. Absolute kinetic theory determines the proton conductivity for the enhancement factor as shown in Figure S7c. ${ }^{44-46}$ In this model we can stimulate that the ionic conductivity can be increased via the modulation of potential energy for the proton transfer between water dimer. From the $\mathrm{OH}$ stretching potential parameters and experimentally observed Rabi splitting values, the increment of the ionic conductivity is observed.

We would like to then discuss the effect of vibrational strong coupling on the aspect of coherence among the molecular vibration of individual water molecules. The cooperative motion of the ionic carrier is important for the hydrogen bonding structurization of water, ${ }^{47}$ and cooperative proton transport. ${ }^{48}$ The coherence in the ionic conductivity is known as the Haven ratio, which is a parameter that governs for the ionic conductivity, which diffusion coefficient from charge transport and diffusion of atoms from random-walk models are compared. ${ }^{49,50}$ In case with the vibrational strong coupling, we can expect the coherent vibration of $\mathrm{OH}$ stretching bond in cavity because the photon exchange is faster than the rate of the vibrational relaxation and cavity leakage. In addition, the Travis-Cumming 
model possesses the formation of the dark state in the cavity and there are some contribution of the dark mode on the charge transport. ${ }^{51-53}$ We assumed that the dark mode in the polaritonic system can contribute to the ion transport, and, therefore, the ionic conductivity can be modulated even for the electrolyte with vehicle mechanism.

We would like to note that the demonstrated study can be, in principle, applied to various ion conductors. Usually, ion conductors have been designed based on the ion binding energy and ionic carrier, ${ }^{54}$ and even nanostructure can be utilized by employing the space-charge layer. ${ }^{55}$ Vibrational structure is ubiquitous for molecular, polymer, and inorganic materials. Various materials can be placed to the optical cavity, and the utilization of oscillating electromagnetic fields in vacuum field enhances the ionic conductivity of materials. The increase of the ionic conductivity can be used for any electrochemical devices, such as water electrolysis, fuel cell, battery systems. The utilization of the vibrational strong coupling can potentially leads to the efficient energy conversion systems by the use of quantum optics principle.

In conclusions, we have discovered that the vibrational strong coupling of water enhances the ionic conductivity. We observed vibrational strong coupling of water electrolyte solutions and the modulation of electrochemical properties dependent on the cavity modes. Theoretical model can be used for the explanation of the observed ionic conductivity and importance of vibrational strong coupling state is indicated. Since vibrational structure is ubiquitous in materials, we envisioned that and inherent promotion of ionic transport is plausible approach via vibrational strong coupling. 


\section{Experimental Methods}

Materials. $\mathrm{Au}, \mathrm{Pt}$ metals are purchased from Tanaka Precious Metals, Japan. $\mathrm{CaF}_{2}$ window was purchased from the Specac Ltd., UK. Mylar films were purchased from the Toray, Dupont Japan. All aqueous electrolyte solutions were prepared with Milli-Q water (Millipore, $18.2 \mathrm{M} \Omega \mathrm{cm}$ resistivity). $\mathrm{D}_{2} \mathrm{O}(99.9 \%)$ was purchased from Sigma-Aldrich.

Preparation of a Fabry-Pérot cavity. A Fabry-Pérot cavity consisting of two parallel mirrors separated by Mylar film was prepared as shown in Figure S1. The mirrors were fabricated by sputtering of $8 \mathrm{~nm}$ of $\mathrm{Au}$ on $2 \mathrm{~mm}$ of $\mathrm{CaF}_{2}$ windows, followed by sputtering of $20 \mathrm{~nm}$ of $\mathrm{SiO}_{2}$. Mirrors were sandwiched with home-made cell, and cavity thickness, $L$, is tuned by screw. Optical cavity modes can be determined by following equations, $\omega_{\text {cav }}(i)=i k_{0}, k_{0}=10^{4}(2 n L)^{-1}$, where $\omega_{\text {cav }}(i)$ is the frequency of the $i$ th optical mode in $\mathrm{cm}^{-1}, i$ is integer, $k_{0}$ is the free spectral range (FSR, corresponding to the peakto-peak separation of cavity modes) in $\mathrm{cm}^{-1}, n$ is the refractive index of the cavity medium, and $L$ is the cavity thickness in $\mu \mathrm{m}$.

IR measurements. IR spectroscopy measurements were conducted by using a JASCO FT-IR 4200. The resolution was set as $4 \mathrm{~cm}^{-1}$ and the spot size was typically $2.5 \mathrm{~mm}$. Quality of cavity cell was confirmed from the infrared spectroscopy and structural color in cavity cell.

Electrochemical impedance measurements. All the electrochemical impedance measurements were conducted by using Biologic VSP-150 potentiostat. Frequency range was set from $1 \mathrm{MHz}$ to $1 \mathrm{~Hz}$. Potential bias was set as $0 \mathrm{~V}$, and amplitude was typically $300 \mathrm{mV}$. For the measurements, two Pt 
electrodes $(\phi=0.3 \mathrm{~mm})$ were introduced to the two holes of window. The ionic conductivity was measured horizonal to the window.

Ionic conductivity and dielectric constants of electrolyte solution were calculated by following equations.

$$
\sigma=\frac{1}{R_{\mathrm{s}}} \frac{d}{A} \quad \varepsilon=C \frac{A}{d}
$$

Where $\mathrm{A}$ is electrode area, $\mathrm{d}$ is electrode distance, respectively. The obtained ionic conductivity or dielectric constant was corrected by using the value obtained from the $\mathrm{CaF}_{2}$. 


\section{References:}

1 Ebbesen, T. W. Hybrid Light-Matter States in a Molecular and Material Science Perspective. Acc Chem Res 49, 2403-2412, doi:10.1021/acs.accounts.6b00295 (2016).

2 Ribeiro, R. F., Martinez-Martinez, L. A., Du, M., Campos-Gonzalez-Angulo, J. \& Yuen-Zhou, J. Polariton chemistry: controlling molecular dynamics with optical cavities. Chem Sci 9, 6325-6339, doi:10.1039/c8sc01043a (2018).

Hertzog, M., Wang, M., Mony, J. \& Borjesson, K. Strong light-matter interactions: a new direction within chemistry. Chem Soc Rev 48, 937-961, doi:10.1039/c8cs00193f (2019). Hutchison, J. A., Schwartz, T., Genet, C., Devaux, E. \& Ebbesen, T. W. Modifying chemical landscapes by coupling to vacuum fields. Angew Chem Int Ed Engl 51, 1592-1596, doi:10.1002/anie.201107033 (2012).

Schwartz, T. et al. Polariton dynamics under strong light-molecule coupling. Chemphyschem 14, 125-131, doi:10.1002/cphc.201200734 (2013).

Orgiu, E. et al. Conductivity in organic semiconductors hybridized with the vacuum field. Nat Mater 14, 11231129, doi:10.1038/nmat4392 (2015).

Hagenmuller, D., Schachenmayer, J., Schutz, S., Genes, C. \& Pupillo, G. Cavity-Enhanced Transport of Charge. Phys Rev Lett 119, 223601, doi:10.1103/PhysRevLett.119.223601 (2017).

Nagarajan, K. et al. Conductivity and Photoconductivity of a p-Type Organic Semiconductor under Ultrastrong Coupling. ACS Nano 14, 10219-10225, doi:10.1021/acsnano.0c03496 (2020).

Shalabney, A. et al. Coherent coupling of molecular resonators with a microcavity mode. Nat Commun 6, 5981, doi:10.1038/ncomms6981 (2015).

10 George, J., Shalabney, A., Hutchison, J. A., Genet, C. \& Ebbesen, T. W. Liquid-Phase Vibrational Strong Coupling. J Phys Chem Lett 6, 1027-1031, doi:10.1021/acs.jpclett.5b00204 (2015).

11 Long, J. P. \& Simpkins, B. S. Coherent Coupling between a Molecular Vibration and Fabry-Perot Optical Cavity to Give Hybridized States in the Strong Coupling Limit. ACS Photonics 2, 130-136, doi:10.1021/ph5003347 (2014).

Du, M., Campos-Gonzalez-Angulo, J. A. \& Yuen-Zhou, J. Nonequilibrium effects of cavity leakage and vibrational dissipation in thermally activated polariton chemistry. $J$ Chem Phys 154, 084108, doi:10.1063/5.0037905 (2021).

13 Thomas, A. et al. Ground-State Chemical Reactivity under Vibrational Coupling to the Vacuum Electromagnetic Field. Angew Chem Int Ed Engl 55, 11462-11466, doi:10.1002/anie.201605504 (2016).

14 Thomas, A. et al. Tilting a ground-state reactivity landscape by vibrational strong coupling. Science 363, 615-619, doi:10.1126/science.aau7742 (2019).

15 Lather, J., Bhatt, P., Thomas, A., Ebbesen, T. W. \& George, J. Cavity Catalysis by Cooperative Vibrational Strong Coupling of Reactant and Solvent Molecules. Angew Chem Int Ed Engl 58, 10635-10638, doi:10.1002/anie.201905407 (2019).

16 Hirai, K., Hutchison, J. A. \& Uji, I. H. Recent Progress in Vibropolaritonic Chemistry. Chempluschem 85, 19811988, doi:10.1002/cplu.202000411 (2020). 

doi:10.1021/ar50053a001 (2002).

18 Eisenberg, D. \& Kauzmann, W. Structure and Properties of Water. (1969).

19 Marx, D., Tuckerman, M. E., Hutter, J. \& Parrinello, M. The nature of the hydrated excess proton in water. Nature 397, 601-604, doi:10.1038/17579 (1999).

20 Ohmine, I. \& Tanaka, H. Fluctuation, relaxations, and hydration in liquid water. Hydrogen-bond rearrangement dynamics. Chemical Reviews 93, 2545-2566, doi:10.1021/cr00023a011 (2002).

21 Auer, B. M. \& Skinner, J. L. IR and Raman spectra of liquid water: theory and interpretation. J Chem Phys $\mathbf{1 2 8 ,}$ 224511, doi:10.1063/1.2925258 (2008).

22 Perakis, F. et al. Vibrational Spectroscopy and Dynamics of Water. Chem Rev 116, 7590-7607, doi:10.1021/acs.chemrev.5b00640 (2016).

23 Grotthuss, C. J. T. Sur la décomposition de l'eau et des corps q'uelle tient en dissolution à l'aide de l'électricité galvanique. Ann. Chim., LVIII, 54-74 (1806).

24 Cukierman, S. Et tu, Grotthuss! and other unfinished stories. Biochim Biophys Acta 1757, 876-885, doi:10.1016/j.bbabio.2005.12.001 (2006).

25 Conway, B. E., Bockris, J. O. M. \& Linton, H. Proton Conductance and the Existence of the H3O·Ion. The Journal of Chemical Physics 24, 834-850, doi:10.1063/1.1742619 (1956).

26 Conway, B. E. \& Bockris, J. O. M. Proton Conductance in Ice. The Journal of Chemical Physics 28, 354-355, doi:10.1063/1.1744127 (1958).

27 Self-dissociation and protonic charge transport in water and. Proceedings of the Royal Society of London. Series A. Mathematical and Physical Sciences 247, 505-533, doi:10.1098/rspa.1958.0208 (1997).

28 Krishtalik, L. I. The mechanism of the proton transfer: an outline. Biochim Biophys Acta 1458, 6-27, doi:10.1016/s0005-2728(00)00057-8 (2000).

29 Kreuer, K. On the complexity of proton conduction phenomena. Solid State Ionics 136-137, 149-160, doi:10.1016/s0167-2738(00)00301-5 (2000).

30 Hidefumi, H., Atef, S. \& Jino, G. Vibrational Ultra Strong Coupling of Water and Ice. (2019).

31 Vergauwe, R. M. A. et al. Modification of Enzyme Activity by Vibrational Strong Coupling of Water. Angew Chem Int Ed Engl 58, 15324-15328, doi:10.1002/anie.201908876 (2019).

32 Li, T. E., Subotnik, J. E. \& Nitzan, A. Cavity molecular dynamics simulations of liquid water under vibrational ultrastrong coupling. Proc Natl Acad Sci U S A 117, 18324-18331, doi:10.1073/pnas.2009272117 (2020).

33 Lather, J. \& George, J. Improving Enzyme Catalytic Efficiency by Co-operative Vibrational Strong Coupling of Water. J Phys Chem Lett 12, 379-384, doi:10.1021/acs.jpclett.0c03003 (2021).

34 Imperatore, M. V., Asbury, J. B. \& Giebink, N. C. Reproducibility of cavity-enhanced chemical reaction rates in the vibrational strong coupling regime. The Journal of Chemical Physics 154, doi:10.1063/5.0046307 (2021).

35 Khitrova, G., Gibbs, H. M., Kira, M., Koch, S. W. \& Scherer, A. Vacuum Rabi splitting in semiconductors. Nature Physics 2, 81-90, doi:10.1038/nphys227 (2006).

36 Torma, P. \& Barnes, W. L. Strong coupling between surface plasmon polaritons and emitters: a review. Reports on Progress in Physics 78, doi:Artn 013901 
10.1088/0034-4885/78/1/013901 (2015).

37 Hertzog, M. \& Börjesson, K. The Effect of Coupling Mode in the Vibrational Strong Coupling Regime. ChemPhotoChem 4, 612-617, doi:10.1002/cptc.202000047 (2020).

38 Stein, D., Kruithof, M. \& Dekker, C. Surface-charge-governed ion transport in nanofluidic channels. Phys Rev Lett 93, 035901, doi:10.1103/PhysRevLett.93.035901 (2004).

39 Tsukahara, T., Hibara, A., Ikeda, Y. \& Kitamori, T. NMR study of water molecules confined in extended nanospaces. Angew Chem Int Ed Engl 46, 1180-1183, doi:10.1002/anie.200604502 (2007).

40 Chinen, H. et al. Enhancement of proton mobility in extended-nanospace channels. Angew Chem Int Ed Engl 51, 3573-3577, doi:10.1002/anie.201104883 (2012).

41 Hubbard, J. \& Onsager, L. Dielectric dispersion and dielectric friction in electrolyte solutions. I. The Journal of Chemical Physics 67, 4850-4857, doi:10.1063/1.434664 (1977).

42 Chandra, A. Static dielectric constant of aqueous electrolyte solutions: Is there any dynamic contribution? The Journal of Chemical Physics 113, 903-905, doi:10.1063/1.481870 (2000).

43 Onsager, L. Electric Moments of Molecules in Liquids. Journal of the American Chemical Society 58, 1486-1493, doi:10.1021/ja01299a050 (2002).

44 Bernal, J. D. \& Fowler, R. H. A Theory of Water and Ionic Solution, with Particular Reference to Hydrogen and Hydroxyl Ions. The Journal of Chemical Physics 1, 515-548, doi:10.1063/1.1749327 (1933).

45 Stearn, A. E. \& Eyring, H. The Deduction of Reaction Mechanisms from the Theory of Absolute Rates. The Journal of Chemical Physics 5, 113-124, doi:10.1063/1.1749988 (1937).

46 Steam, A. E., Irish, E. M. \& Eyring, H. A Theory of Diffusion in Liquids. The Journal of Physical Chemistry 44, 981-995, doi:10.1021/j150404a002 (2002).

47 Geissler, P. L., Dellago, C., Chandler, D., Hutter, J. \& Parrinello, M. Autoionization in liquid water. Science 291, 2121-2124, doi:10.1126/science.1056991 (2001).

48 Vilciauskas, L., Tuckerman, M. E., Bester, G., Paddison, S. J. \& Kreuer, K. D. The mechanism of proton conduction in phosphoric acid. Nat Chem 4, 461-466, doi:10.1038/nchem.1329 (2012).

49 Murch, G. The haven ratio in fast ionic conductors. Solid State Ionics 7, 177-198, doi:10.1016/01672738(82)90050-9 (1982).

50 Dippel, T. \& Kreuer, K. Proton transport mechanism in concentrated aqueous solutions and solid hydrates of acids

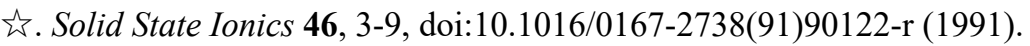

51 Vurgaftman, I., Simpkins, B. S., Dunkelberger, A. D. \& Owrutsky, J. C. Negligible Effect of Vibrational Polaritons on Chemical Reaction Rates via the Density of States Pathway. J Phys Chem Lett 11, 3557-3562, doi:10.1021/acs.jpclett.0c00841 (2020).

52 Scholes, G. D., DelPo, C. A. \& Kudisch, B. Entropy Reorders Polariton States. J Phys Chem Lett 11, 6389-6395, doi:10.1021/acs.jpclett.0c02000 (2020).

53 Botzung, T. et al. Dark state semilocalization of quantum emitters in a cavity. Physical Review B 102, doi:ARTN 144202

10.1103/PhysRevB.102.144202 (2020).

54 Kreuer, K.-D. Proton Conductivity: Materials and Applications. Chemistry of Materials 8, 610-641, 
doi:10.1021/cm950192a (1996).

55 Maier, J. Nanoionics: ion transport and electrochemical storage in confined systems. Nat Mater 4, 805-815, doi:10.1038/nmat1513 (2005). 


\section{Acknowledgements:}

This work was supported by JSPS KAKENHI Grant Number JP21K14596 and JP20H05281 (Grant-in-Aid for Scientific Research on Innovative Areas "Interface IONICS"). This work was partially supported JSPS KAKENHI Grant Number JP16H06506 (Grant-in-Aid for Scientific Research on Innovative Areas "Optical manipulation”). Support by the Photo-excitonix Project in Hokkaido University is also acknowledged. 